\title{
Spectrum of HIV Associated Malignancies: A Retrospective Study of 42 Patients over a Period of 16 Years Attending a Tertiary Care Hospital
}

\author{
Rajendra Kumar Tanwar ${ }^{1, \text { * }}$, Bharti Saxena ${ }^{2}$, Manu Singh Tanwar ${ }^{3}$, Abhirup Chatterjee ${ }^{4}$, \\ Harsh Goyal $^{1}$, Naveen Saxena ${ }^{5}$, Raghuveer Singh ${ }^{6}$, Madhu Saxena ${ }^{7}$, Uma Shankar Shukla ${ }^{6}$ \\ ${ }^{1}$ Department of Radiation Oncology, Rajasthan University of Health Sciences, Jaipur, India \\ ${ }^{2}$ Department of Obstetrics and Gynecology, Rajasthan University of Health Sciences, Jaipur, India \\ ${ }^{3}$ Department of Conservative Dentistry \& Endodontics, Maharishi Markandeshwar University, Ambala, India \\ ${ }^{4}$ Department of General Dentistry, Rajasthan University of Health Sciences, Jaipur, India \\ ${ }^{5}$ Department of Microbiology, Rajasthan University of Health Sciences, Jaipur, India \\ ${ }^{6}$ Department of Community Medicine, Rajasthan University of Health Sciences, Jaipur, India \\ ${ }^{7}$ Department of Anesthesiology, Rajasthan University of Health Sciences, Jaipur, India
}

\section{Email address:}

rktanwar@rediffmail.com (R. K. Tanwar), bharti.saxena.kota@gmail.com (B. Saxena), singhtanwarmanu@gmail.com (M. S. Tanwar), Dr.abhi.maxfac@gmail.com (A. Chatterjee),drharsh222@gmail.com (H. Goyal),drnaveen_saxena@yahoo.co.in (N. Saxena), singhraghuveer2008@gmail.com (R. Singh),rktanwar@rediffmail.com (M. Saxena),drusshuklapcms@gmail.com (U. S. Shukla)

${ }^{*}$ Corresponding author

\section{To cite this article:}

Rajendra Kumar Tanwar, Bharti Saxena, Manu Singh Tanwar, Abhirup Chatterjee, Harsh Goyal, Naveen Saxena, Raghuveer Singh, Madhu Saxena, Uma Shankar Shukla. Spectrum of HIV Associated Malignancies: A Retrospective Study of 42 Patients over a Period of 16 Years Attending a Tertiary Care Hospital. Cancer Research Journal. Vol. 9, No. 3, 2021, pp. 131-139. doi: 10.11648/j.crj.20210903.11

Received: June 15, 2021; Accepted: June 30, 2021; Published: July 7, 2021

\begin{abstract}
Introduction: The Human immunodeficiency virus (HIV) infection remains a serious public health concern in India and around the world at large. Malignancy is frequent among people living with HIV (PLWH) has become the leading cause of death. The incidence of malignancy among PLWH depends on various factors; virological control under combined antiretroviral therapy (cART), the exposure to oncogenic virus is of utmost importance, which can be prevented with the implementation of specific screening programs. Drug-drug interactions between cART and oncologic treatments can lead to serious adverse effects or to a reduction in the therapeutic effects requiring close monitoring. Methods: This is a single center retrospective study conducted on 42 seropositive patients attending a tertiary care oncology department from July 2005 to June 2021 to assess the demographic profile; laboratory investigations, clinico-pathological correlation, treatment outcome and survival follow up. Results: A total of 26410 patients were registered in the department during this period, out of which 42 $(0.16 \%)$ were found to be HIV positive. These included head \& neck -13; uterine cervix-7; lung -4; esophagus-4; NHL-4; recto sigmoid -3; breast-3; gall bladder; endometrium, choriocarcinoma (CCA) and acute myeloid leukemia (AML) one each. Conclusion; Seropositive patients often present in advanced stage of disease but have a good prognosis if treated appropriately with anti-retroviral therapy (ART). Very few studies have been published in India regarding the incidence of malignancy in HIV patients credited as one of the largest study published till date.
\end{abstract}

Keywords: HIV, NADC, ADC, Kaposi Sarcoma, NHL, Oral Squamous Cell Carcinoma, Immune Checkpoint Inhibitor

\section{Introduction}

Acquired immune deficiency syndrome (AIDS) was first recognized in U.S. in 1981, when Centers for Disease
Control and Prevention (CDC) reported the unexplained occurrence of Pneumocystis Jirovecii pneumonia in 5 previously healthy homosexual men in Los Angeles; and the occurrence of Kaposi's sarcoma with or without 
Pneumocystis Jirovecii pneumonia and other opportunistic infections in 26 previously healthy homosexual men in New York, San Francisco and Los Angeles. [1] In 1983, the HIV virus was first isolated from a patient with lymphadenopathy and by 1984 , it was demonstrated clearly to be causative agent of AIDS. In May 1986, the International Committee on Taxonomy gave it a new name "HIV". [2] In 1985, ELISA test was developed. [2] AIDS is diagnosed when an individual has a CD-4 T cell lymphocyte count $<200 / \mathrm{mm}^{3}$ and suffers from opportunistic infections because of suppressed immune system. The immunosuppression is measured by $\mathrm{CD}-4$ T-cell counts. People with healthy immune system usually have $>95 \%$ CD-4 T-cells $/ \mu \mathrm{L}$ of blood. When Lymphocyte count is $<14 \%$, the risk of progression to AIDS is high. The route of transmission is heterosexuality $(88.2 \%)$, parent to child $(5 \%)$, injectable drug abusers $(1.7 \%)$, homosexuals $(1.5 \%)$, patients receiving blood and blood products (1\%) and unknown etiology $(2.7 \%)$. [3] The virus has been found in highest concentration in blood, semen and CSF. Lower concentration has been detected in tears, breast milk, urine, cervical and vaginal secretions. The virus has been isolated from brain tissue, lymph node, buccal mucosa and skin. The ELISA test is sensitive to detect HIV antibodies while Western Blot test is confirmatory and highly specific for the same.

There are certain cancers that are recognized to affect people infected with HIV; referred to as AIDS defining cancers (ADC). These include: 1. Kaposi sarcoma, 2. Invasive cervical carcinoma and 3. NHL including primary CNS lymphoma. However, there are other types of cancers that appear more among HIV infected people compared with the general population but were not previously found associated with HIV infection. These are Non AIDS defining cancers (NADC) which include anal, lung [non small cell lung carcinoma (NSCLC)], liver (hepatocellular carcinoma), kidney, testicular, Hodgkin's Lymphoma (HL), head and neck cancers (HNC), multiple myeloma, brain, gastric and other cancers. [4] With the introduction of highly active antiretroviral therapy (HAART), a large number of patients with HIV are surviving for a longer time thus PLWH have a higher incidence of many cancers compared with the general population with multiple contributing factors including a higher chance of co-infection with oncogene virus such as Human papilloma virus (HPV) - (cancer cervix, vulva, anus, penile warts and head and neck cancers), Epstein Barr virus (EBV) - (Burkitt's lymphoma, HL, nasopharyngeal carcinoma, B-cell Lymphoma), Hepatitis B Virus (HBV) and Hepatitis C Virus (HCC) - (primary hepatocellular cancer), Human herpes virus -8 (HHV-8) - (Kaposi sarcoma and Primary effusion lymphoma), Human T-cell leukemia virus (HTLV-1) - (T-cell leukemia, lymphoma and primary effusion lymphoma). In addition to this, non-viral co-factors are smoking and alcohol consumption. The smoking and alcohol are strongly synergistic risk factors. [5] In AIDS patients the risk of developing malignancy is higher than in non HIV population, cancer cervix being 5-7 times, anal cancer 13 times [6], cancer lung 5-7 times and Hodgkin's lymphoma 5-14 times, NHL 10-20 times [7], head and neck 3 times and leukemia 2.5 times. [8] No increased risk was observed for breast, prostate and colorectal cancers in the HIV positive cohort. [9]

In the past, the PLWH were excluded from the oncologic clinical research trials with the aim of assessing the efficacy and toxicity profile of the immune check point inhibitors (ICPi) because of an increased theoretical risk of inducing adverse events and a feared decreased efficacy in the immune compromised population. However, most of the retrospective clinical data reporting the use of ICPi among PLWH are somewhat reassuring with a safety and efficacy profile similar to that observed in HIV-negative patients. Regarding the "shock and kill" anti-HIV effects of ICPi, the preliminary clinical data available are still modest and relatively disappointing despite encouraging results obtained in vitro. HIV-associated cancers represent a particular care challenge to the oncologist due to the multiple comorbidities in this population and the high risk of drug interactions. Thus the CANCER VIH (working group in a National French multidisciplinary Network dedicated to HIV infected patients) is of particular interest in this context. HIV patients who are diagnosed as malignancy are considered WHO clinical stage IV of HIV. The malignancy occurs 1-2 decades earlier in AIDS patients. WHO strongly recommends initiating ART for all PLWH regardless of CD-4 $+\mathrm{T}$ cell count, after two large randomized trials demonstrated overall benefits especially in terms of non AIDS defining morbidities and mortalities. [10]

The life expectancy on ART is gradually increasing and higher burden of viral oncogene caused HIV associated malignancy (HIVAM) to become the second leading cause of deaths after tuberculosis (TB) in PLWH. The risk of developing Kaposi sarcoma had declined significantly (up to $90 \%$ ) with use of ART. Hence ART is really the backbone of treatment of Kaposi Sarcoma. Hence all cancer patients should be screened for HIV prior to commencement of treatment.

\section{Methods}

A retrospective review of medical records of 42 patients diagnosed to have HIV and malignancy from July 2005 to June 2021 i.e. over a period of 16 years (total 26410 patients) who visited the department of Radiation Oncology in our tertiary care hospital was performed. The records were evaluated for demographic profile, clinicopathological presentation, routine hematological, serological tests including hepatic, renal, Australia antigen, HIV, CD-4 count, thyroid function test, skiagram of chest, ultrasonography of abdomen and pelvis were done. Computerized Tomography (CT) scan was done to evaluate the clinical staging of the disease. Eastern Co-operative Oncology Group (ECOG) performance status was noted. Clinical staging of all head and neck cancer patients was done according to AJCC staging; gynecological patients according to FIGO staging. All patients were discussed in tumor board and their 
treatment decided. Treatment related toxicities were noted and managed accordingly. All patients were advised to visit ART clinic and take ART along with treatment of cancer regularly as per guidelines. CD-4 count was done periodically in all patients. After treatment of malignancy, patients were followed up regularly till death.

\section{Results}

The ART center at our medical college, hospital was started in 2009 and till June 2021, a total of 4516 patients were registered, out of these only 42 patients $(0.93 \%)$ were diagnosed to have malignancy. In my department 26410 patients registered from July 2005 to June 2021 and out of these, 42 patients $(0.16 \%)$ were diagnosed to have AIDS. A large number of our patients presented with advanced stage of the disease due to a number of reasons. Majority of them were illiterate, belonging to poor socioeconomic status, hence they reached the oncology treatment center quite late. We observed ADC (cancer cervix and NHL) were 11 and NADC were 31 . The age ranged between 32-70 years (Mean 44.7 years). Sex distribution showed males 23; females 19 and $\mathrm{M}$ : F ratio 1.21: 1 (Table 1). In 36 patients, CD-4 count was available and average CD-4 count was 391. Regarding investigations - Hb below $10 \mathrm{Gm} \%$ in 4; deep jaundice in 1 and renal failure in 3 patients. Regarding clinical presentation lymphadenopathy in 18 (all 13 head and neck malignancy, 2 lung, 2 breast and I cervix cancer patients), hepatomegaly with metastasis 6 , splenomegaly with metastasis 2 , pleural effusion 4, lung metastasis 7 , bone metastasis 6 , brain metastasis in 2 and Covid-19 in patient of endometrial carcinoma at the time of presentation. Incidental hypothyroidism was detected in 3 patients. All 4 lung cancer patients presented with advanced disease, 2 with pleural effusion, 1 each with liver, bone and brain metastasis (Table 3). Three patients of carcinoma esophagus presented with upper esophageal disease and interestingly one patient presented with situs inversus totalis (SIT) who developed hepatic metastasis and died soon after completion of radiation therapy treatment. [11] (Table 3) NHL breast patient was a beggar who was accompanied by a blind fellow who used to sell newspapers and magazines in the running trains. [12] (Table 4) One cancer breast patient presented with synchronous bilateral disease with distant metastasis. One breast cancer patient's mother and aunt were also treated for breast cancer in the past (Table 4). The serology of HIV test revealed HIV I \& II in 33 and HIV I in 9 patients (Tables 25), Australia antigen test was reactive in 1 and non-reactive in 41 patients. Spouses of 14 patients were found to be HIV positive, 20 were negative and status of 8 was not known (Tables 2-5). The children of all patients were seronegative. The recto sigmoid gastrointestinal stromal tumor (GIST) was diagnosed in 2005, she developed intestinal obstruction in 2012 and intra operatively 6 units of blood were transfused. She was found HIV positive in 2016 after this blood transfusion (Table 3). In 4 patients anti-tubercular treatment (3 pulmonary and 1 extra pulmonary) was given (Tables 2, 3, 5). All 3 gastrointestinal malignancies were either sigmoid or recto sigmoid in origin. Two gastro intestinal and 1 gall bladder malignancy had disseminated abdominal disease at presentation while one presented with intestinal obstruction (Table 3). One patient of carcinoma alveolus developed second primary malignancy (SPM) 7 years after primary treatment (Table 2). One newly diagnosed patient with endometrial carcinoma had Covid-19 disease and died without anti cancer treatment (Table 4). The number of patients in whom diagnosis of HIV, institution of ART and diagnosis of malignancy are shown in figure 1.

Follow Up - All patients were examined during follow-up visits for opportunistic infections like oral thrush, herpes zoster, herpes simplex and other cutaneous lesions, sore throat, weight loss, fever, anemia and evidence of mycobacterium tuberculosis infection and status of metastasis.

Table 1. Age distribution in present study.

\begin{tabular}{lll}
\hline S. N. & Age group & No. of Patients \\
\hline 1 & $31-40$ & 13 \\
2 & $41-50$ & 17 \\
3 & $51-60$ & 7 \\
4 & $61-70$ & 5 \\
\hline
\end{tabular}

Table 2. Profile of Head \& Neck Malignancies.

\begin{tabular}{|c|c|c|c|c|c|c|c|c|}
\hline S. N. & Cancer & Age \& Sex & Diagnosis of HIV & HIV I/II & CD4 Average & Spouse & ART Started & Diagnosed Cancer \\
\hline 1 & Gingivo Buccal Sulcus & $34 \mathrm{M}$ & Oct-09 & I \& II & 380 & Positive & Oct-09 & Jun-13 \\
\hline 2 & Tonsil & $50 \mathrm{~F}$ & May-13 & I \& II & 386 & - & May-13 & May-13 \\
\hline 3 & Tonsil & $39 \mathrm{~F}$ & Jun-13 & I \& II & 428 & - & Jun-13 & Jun-13 \\
\hline 4 & PFS & $58 \mathrm{M}$ & Jun-15 & I \& II & 737 & Negative & Mar-19 & Feb-19 \\
\hline 5 & Buccal Mucosa & $42 \mathrm{M}$ & Aug-15 & I \& II & 620 & - & Aug-15 & Sep-20 \\
\hline 6 & Laryngo pharynx & $49 \mathrm{M}$ & Nov-15 & I \& II & 395 & Positive & Nov-15 & Dec-16 \\
\hline 7 & Vallecula & $50 \mathrm{M}$ & Mar-16 & I \& II & 520 & Negative & Mar-16 & Jan-21 \\
\hline 9 & Base of tongue & $62 \mathrm{M}$ & Apr-18 & I \& II & - & Negative & Apr-18 & Apr-18 \\
\hline 10 & Hard Palate & $45 \mathrm{M}$ & Feb-19 & I \& II & 320 & Negative & Feb-19 & Feb-19 \\
\hline 11 & Alveolus & $44 \mathrm{M}$ & Oct-09 & I \& II & 595 & Negative & Oct-09 & Jun-13 \\
\hline 12 & Tonsil & $56 \mathrm{~F}$ & Jul-20 & I \& II & 380 & Negative & Jul-20 & Jul-20 \\
\hline 13 & Pyriform sinus & $70 \mathrm{M}$ & Oct-20 & I \& II & 423 & Positive & Oct-20 & Nov-20 \\
\hline
\end{tabular}


Table 2. Continued.

\begin{tabular}{llllllll}
\hline S. N. & FNAC/BIOPSY & Staging of Cancer & Treatment Given & Survival of AIDS & Survival of Cancer & Comment & Remark \\
\hline 1 & WDSCC & IV & RT & 48 Months & 6 Months & LFU & - \\
2 & WDSCC & IV & RT & 6 Months & 6 Months & LFU & - \\
3 & MDSCC & IV & RT & 7 Months & 7 Months & LFU & - \\
4 & PD Ca & IV & RT & 54 Months & 6 Months & LFU & - \\
5 & WDSCC & IV & RT & 66 Months & 7 Months & LFU & - \\
6 & MDSCC & IV & RT & 19 Months & 7 Months & LFU & Auto Driver \\
7 & MDSCC & IV & RT & 64 Months & 6 Months & LFU & - \\
8 & WDSCC & IV & RT & 6 Months & 6 Months & LFU & - \\
9 & MDSCC & IV & RT & 5 Months & 5 Months & LFU & - \\
10 & WDSCC & IV & RT & 6 Months & 6 Months & LFU & - \\
11 & WDSCC & II & SUR + RT & 126 Months & 99 Months & SPM 2020 & RT \\
12 & PD Ca & IV & RT & 9 Months & 9 Months & LFU & - \\
13 & WDSCC & IV & RT Months & 5 Months & LFU & ATT \\
\hline
\end{tabular}

WDSCC -well differentiated squamous cell carcinoma

MDSCC-moderately differentiated squamous cell carcinoma-

PDSCC-poorly differentiated squamous cell carcinoma

SPM- second primary malignancy

RT- radiation therapy

SUR- surgery

LFU- Lost to follow up

ATT-anti tubercular treatment

BT- blood transfusion

Table 3. Profile of Lung, Esophagus and GI Malignancies.

\begin{tabular}{lllllllll}
\hline S. No. & Cancer & Age \& Sex & Diagnosis of HIV & HIV I/II & CD4 Average & Spouse & ART Started & Diagnosed Cancer \\
\hline 1 & Lung & $35 \mathrm{~F}$ & Feb-10 & I \& II & 345 & Positive & Jun-19 & Dec-20 \\
2 & Lung & $60 \mathrm{M}$ & Jul-18 & I \& II & 747 & Negative & Jul-18 & Jul-18 \\
3 & Lung & $62 \mathrm{M}$ & Jul-19 & I \& II & 131 & Positive & Jul-19 & Nov-20 \\
4 & Lung & $65 \mathrm{M}$ & Jan-20 & I & - & Negative & Jan-20 & Jan-20 \\
5 & Esophagus & $35 \mathrm{M}$ & Jul-12 & I \& II & 460 & Negative & Jul-12 & Aug-13 \\
6 & Esophagus & $35 \mathrm{M}$ & Oct-12 & I \& II & 380 & Negative & Oct-12 & Sep-13 \\
7 & Esophagus & $50 \mathrm{~F}$ & Mar-15 & I \& II & - & Negative & Mar-15 & Mar-15 \\
8 & Esophagus & $55 \mathrm{M}$ & Sep-15 & I \& II & 200 & Negative & Sep-15 & Oct-16 \\
9 & Sigmoid & $38 \mathrm{M}$ & Jul-13 & I \& II & 513 & Negative & Jul-13 & Apr-16 \\
10 & GIST Sigmoid & 58 & Apr-16 & I & 294 & Negative & Apr-16 & Sep-05 \\
11 & Gall Bladder & $50 \mathrm{M}$ & Jan-16 & I & 552 & Positive & Jan-16 & Jan-16 \\
12 & Recto sigmoid & $42 \mathrm{M}$ & Nov-17 & I & 259 & Negative & Nov-17 & Nov-17 \\
\hline
\end{tabular}

Table 3. Continued.

\begin{tabular}{llllllll}
\hline S. No. & FNAC/BIOPSY & Staging of Cancer & Treatment Given & Survival of AIDS & Survival of Cancer & Comment & Remark \\
\hline 1 & WDSCC & IV & Nil & 120 Months & 4 Months & LFU & \\
2 & WDADC & IV & Chemotherapy & 66 Months & 6 Months & LFU & ATT Given, Driver \\
3 & PD Ca & IV & Cranial RT & 18 Months & 2 Months & LFU & brain Metastasis, ATT \\
4 & WDSCC & IV & Nil & 2 Months & 2 Months & LFU & Disseminated Disease \\
5 & MDSCC & III & RT & 21 Months & 10 Months & LFU & Photographer \\
6 & MDSCC & IV & RT & 14 months & 3 Months & Died & SIT, TB \\
7 & WDSCC & III & RT & 4 months & 4 Months & LFU & Pulm TB \\
8 & MDSCC & III & RT & 15 Months & 4 Months & LFU & - \\
9 & WDADC & IV & RT & 38 months & 4 Months & LFU & Disseminated Disease \\
10 & GIST & III & SUR +IMATINIB & 60 Months & 186 Months & Alive & Blood Transfusion, HZ \\
11 & ADC & IV & Nil & 3 Months & 3 Months & LFU & HZ \\
12 & MDADC & III & SUR + RT + CHEMO & 29 Months & 29 Months & Died & Intestinal Obstruction \\
\hline
\end{tabular}

WDSCC -well differentiated squamous cell carcinoma

MDSCC-moderately differentiated squamous cell carcinoma-

PDSCC- poorly differentiated squamous cell carcinoma

SPM- second primary malignancy

RT- radiation therapy

SUR- surgery

LFU- Lost to follow up

ATT-anti tubercular treatment

BT- blood transfusion

SIT- situs inversus totalis

GIST-gastrointestinal stromal tumor; TB- tuberculosis; HZ-Herpez zoster 
Table 4. Profile of Gynecologic and Breast Malignancies.

\begin{tabular}{lllllllll}
\hline S. No. & Cancer & Age \& Sex & Diagnosis of HIV & HIV I/II & CD4 Average & Spouse & ART Started & Diagnosed Cancer \\
\hline 1 & Cervix & $34 \mathrm{~F}$ & Sep-12 & I \& II & 360 & Positive & Sep-12 & Jun-13 \\
2 & Cervix & $43 \mathrm{~F}$ & Mar-13 & I \& II & 380 & Positive & Mar-13 & Mar-13 \\
3 & Cervix & $39 \mathrm{~F}$ & Oct-16 & I \& II & 260 & - & Oct-16 & Oct-16 \\
4 & Cervix & $40 \mathrm{~F}$ & Aug-17 & I \& II & 320 & Positive & Aug-17 & Aug-17 \\
5 & Cervix & $35 \mathrm{~F}$ & Dec-17 & I & 485 & Positive & Dec-17 & Dec-17 \\
6 & Cervix & $57 \mathrm{~F}$ & Mar-20 & I & 380 & - & Jun-20 & Mar-20 \\
7 & Cervix & $55 \mathrm{~F}$ & Jun-21 & I \& II & - & Positive & Jun-21 & Jun-21 \\
8 & CCA & $32 \mathrm{~F}$ & Dec-12 & I \& II & 480 & - & Dec-12 & Dec-12 \\
9 & Endometrium & $45 \mathrm{~F}$ & Sep-14 & I & 595 & Positive & Sep-17 & April 21 \\
10 & Breast & $42 \mathrm{~F}$ & Dec-15 & I \& II & 520 & Positive & Dec-15 & Aug-20 \\
11 & Breast & $45 \mathrm{~F}$ & Jul-16 & I \& II & 65 & Negative & Jul-16 & Jul-16 \\
12 & Breast & $45 \mathrm{~F}$ & Jun-21 & I & - & - & Jun-21 & Jun21 \\
\hline
\end{tabular}

Table 4. Continued.

\begin{tabular}{|c|c|c|c|c|c|c|c|}
\hline S. No. & FNAC/BIOPSY & $\begin{array}{l}\text { Staging of } \\
\text { Cancer }\end{array}$ & $\begin{array}{l}\text { Treatment } \\
\text { Given } \\
\end{array}$ & $\begin{array}{l}\text { Survival of AIDS } \\
\text { (Months) }\end{array}$ & $\begin{array}{l}\text { Survival of } \\
\text { Cancer (Months) } \\
\end{array}$ & Comment & Remark \\
\hline 1 & MDSCC & IIIB & RT & 22 & 12 & LFU & Husband Driver \\
\hline 2 & MDSCC & IIIB & RT & 6 & 6 & LFU & - \\
\hline 3 & MDSCC & IIIB & RT & 8 & 8 & LFU & - \\
\hline 4 & LCKSCC & IIIB & RT & 7 & 7 & LFU & - \\
\hline 5 & WDKSCC & IV & RT & 40 & 40 & Died & - \\
\hline 6 & MDSCC & IV A & RT & 8 & 8 & LFU & - \\
\hline 8 & $\mathrm{CCA}$ & IV & SUR + EMACO & 100 & 100 & Alive & - \\
\hline 9 & MDADC & IIB & - & 79 & - & Died Covid-19 & - \\
\hline 10 & IDC & IV & SUR + CHEMO & 64 & 8 & Alive on Treatment & Cable Operator \\
\hline 11 & IDC & IV & SUR + CHEMO & 4 & 4 & LFU & - \\
\hline 12 & IDC & IV & Tamoxifen & - & - & On treatment & - \\
\hline
\end{tabular}

WDSCC -well differentiated squamous cell carcinoma

MDSCC- moderately differentiated squamous cell carcinoma-

PDSCC- poorly differentiated squamous cell carcinoma

IDC-infiltrative duct carcinoma

CCA-choriocarcinoma

RT- radiation therapy

SUR- surgery

LFU- Lost to follow up

BT- blood transfusion

EMACO-Etoposide + methotrexate + actinomycin-D + cyclophosphamide + vincristine

Table 5. Profile of Hematologic Malignancies.

\begin{tabular}{lllllllll}
\hline S. No. & Cancer & Age \& Sex & Diagnosis of HIV & HIV I/II & CD4 Average & Spouse & ART Started & Diagnosed Cancer \\
\hline 1 & NHL Breast & $61 \mathrm{~F}$ & Oct-10 & I \& II & 465 & Unmarried & Oct-10 & Sep-16 \\
2 & NHL SKULL & $38 \mathrm{M}$ & Dec-12 & I \& II & 440 & Negative & Dec-12 & Dec-12 \\
3 & NHL & $44 \mathrm{M}$ & Jan-18 & I \& II & - & Negative & Jan-18 & Jan-18 \\
4 & NHL Intestine & $35 \mathrm{M}$ & Mar-19 & I \& II & 85 & Positive & Mar-19 & Aug-20 \\
5 & AML -M2 & $43 \mathrm{M}$ & Nov-06 & I \& II & 360 & Negative & Nov-13 & Sep-19 \\
\hline
\end{tabular}

Table 5. Continued.

\begin{tabular}{llllllll}
\hline S. No. & FNAC/BIOPSY & Staging of Cancer & Treatment Given & Survival of AIDS & Survival of Cancer & Comment & Remark \\
\hline 1 & NHL -DLBCL & I & CHOP 6 Cycles & 92 months & 21 months & - & Bagger \\
2 & NHL -DLBCL & II & CHOP 6 Cycles & 100 Months & 100 Months & - & Tailor \\
3 & NHL -DLBCL & IV & Nil & 3 Months & 3 months & - & - \\
4 & NHL -DLBCL & IV & CHOP 6 Cycles & 12 Months & 9 Months & - & Antik \\
5 & AML & AML M-2 & Palliative Chemo & 157 Months & 3 Months & LFU & - \\
\hline
\end{tabular}

LFU- Lost to follow up

ATT-anti tubercular treatment

BT- blood transfusion

DLBCL-diffuse large B-cell lymphoma

CHOP-cyclophosphamide + doxorubicin + vincristine + prednisolone 


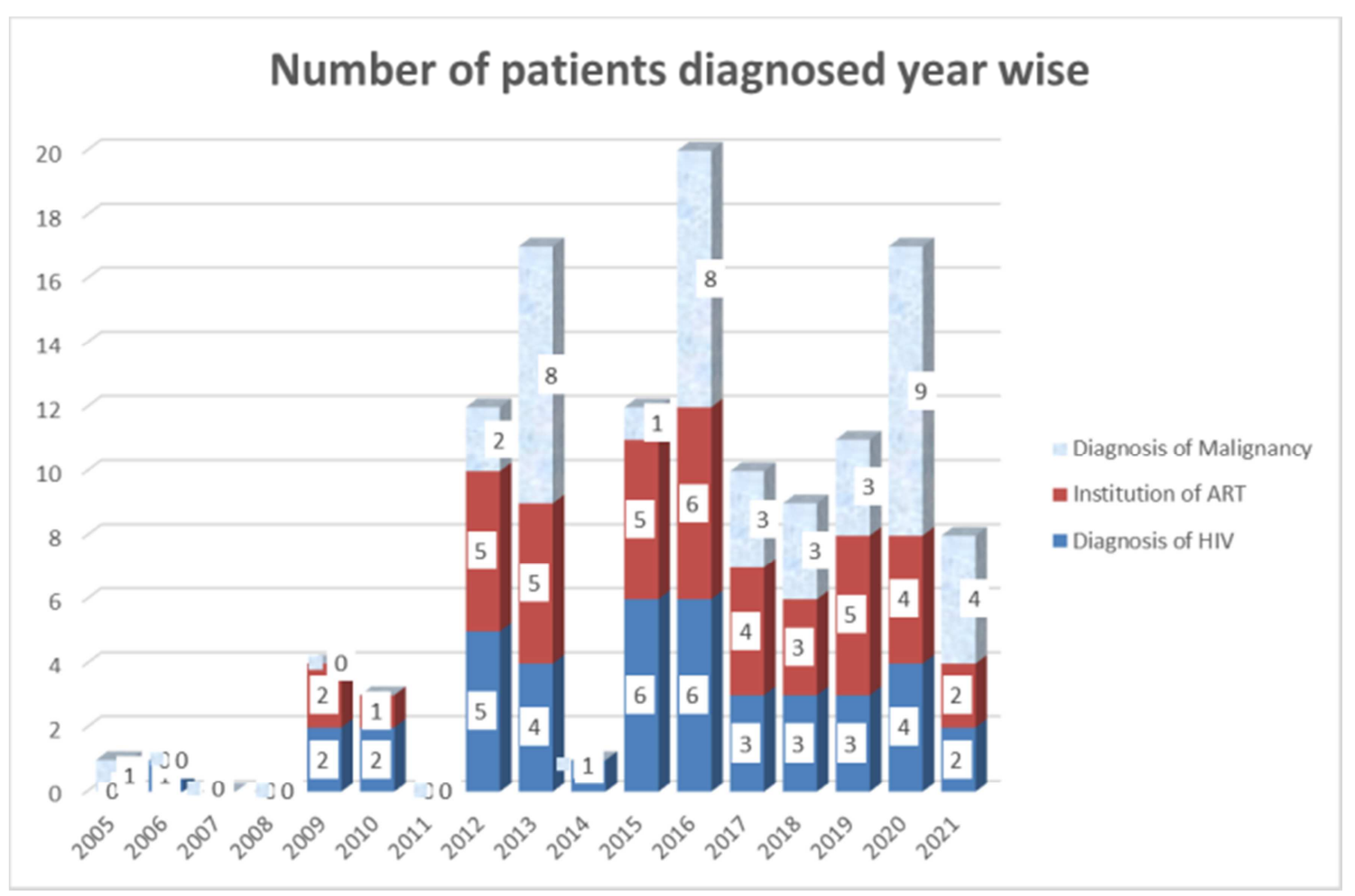

Figure 1. Shows the number of patients in whom diagnosis of HIV, institution of ART and diagnosis of malignancy are shown.

\section{Discussion}

In general, life time risk of developing cancer in PLWH receiving ART remains $25-40 \%$, whereas malignancy accounts for approximately $33 \%$ of all HIV related deaths. [8] The treatment decisions require an understanding of the patients HIV disease as well as curative potential of the malignancy encountered. The treatment outcome of PLWH and malignancy has improved in the past two decades. PLWH have decreased tolerance to chemo radiotherapy compared to those without HIV. Hematologic toxicities results in dose reduction or elimination of concurrent chemotherapy during radiotherapy. [13, 14] Therefore, there is need for novel therapy for management of virally mediated tumors in persons with HIVAM due to advanced immunosuppression and how to co-manage both diseases in ARV- naïve persons. Data on the HIV viral load are lacking in all our patients and CD-4 T-cell counts at the time of diagnosis are available in 36 out of 42 patients. HIV related cancers are preventable through vaccination and early institution of HAART. Screening of all HIV patients with clinical and radiological examination should be conducted for early diagnosis and treatment of cancers.

The head and neck squamous cell carcinoma (HNSCC) are among the most common cancers in Southeast Asia [15]. Oral cavity cancers are most common because of rampant use of chewable tobacco, areca nut, alcohol consumption and HPV infection in general population. [16] These malignancies exhibit profound immunosuppression, aggressive behavior and advanced disease at diagnosis which is responsible for poor treatment outcome. [15]
Treatment complications such as secondary candidiasis and oral mucositis were more common and severe in HIV positive patients and the treatment outcome was significantly worse. [5] Purgina B in his study analyzed 207 cases of HNC cancers and found that $32 \%$ had oropharyngeal, 14\% had tonsillar, $8.69 \%$ had laryngeal and oral cavity and $38.46 \%$ had lip cancers. [5] Zwave NB, in his analysis of 389 patients of $\mathrm{HNC}$, found oral squamous cell carcinoma (OSCC) in $18.8 \%$ cases. [4] In the present study OSCC is reported in $10 \%$ patients.

Cervical cancer is one of the most common cancers worldwide with about 5 lac new cases and 2.7 lac deaths per year. [17] HPV plays animportant role in the etiology of cervical cancer. There are over 80 different HPV sub types. Only HPV 16 and 18 are responsible for most of the neoplastic lesions. HIV induced immune deficiency increases the risk of cancer with a permissive mechanism, promoting the accumulation of genetic damage and progression from dysplasia to cancer. [17] Treatment of these patients is like their seronegative counterparts. HAART should be started / continued. Patients on HAART reported better treatment compliance and manageable toxicities. Wertheim's hysterectomy is done in operable cases. Radiation therapy including teletherapy and brachytherapy and chemotherapy should be given to these patients. Grover S. observed that low CD-4 T-cell count carries more morbidities and mortalities after chemo irradiation treatment. [18] Palliative radiotherapy fractionation schedules are effective for patients with poor performance status and locally advanced disease in relieving the symptoms related to the cervix. An emphasis should be given to the increased mucosal and skin toxicities and to improve compliance and clinical outcome. Several 
studies show a predominance of advanced disease at the time of diagnosis in these patients as compared to general population and a poorer treatment outcome. [17, 19] Our observations also yield similar results.

Uterine Carcinoma: Only 7 cases of endometrial carcinoma are reported in the literature. [20] Of these 7 women, 5 were diagnosed as stage IA endometrial cancer, and all 5 underwent surgery with lymph node assessment. One woman with advanced disease underwent staging laparotomy followed by adjuvant chemotherapy. One woman received only 1 cycle of chemotherapy and died of complications. Ours is the $8^{\text {th }}$ case who presented with COVID-19 illness and died of the same disease. To our knowledge, there is no case series describing specific guidelines, care and outcome of HIV infected women with gynecologic cancers.

Choriocarcinoma: CCA is an aggressive form of gestational trophoblastic neoplasia (GTN) that carries poor prognosis owing to severe immunosuppression, poor performance status, sepsis and extensive metastases. Early deaths are attributed to respiratory compromise, hemorrhage secondary to heavy tumor burden within the thorax, and rapid tumor lysis associated with full-dose chemotherapy treatment. Hence, the introduction of low-dose chemotherapy is the initial treatment of high-risk patients to gradually reduce tumor volume and significantly reduce the risk of early death. Only 4 cases of CCA with AIDS have been reported in the literature till now. [21] Ours is the $5^{\text {th }}$ case report which demonstrates the successful management of choriocarcinoma in an HIV-infected patient. [22]

Lung cancer is a major cause of increased morbidity and mortality in the HIV infected persons. As excessive smoking leads to chronic inflammation and COPD, and HIV causes immunosuppression, hence HIV infected smokers appear to develop lung cancer at an earlier age than their non HIV infected counterparts. [23] Cessation of smoking may be safe (prophylactic) and detection of lung cancer using CT scan chest may help in early detection of lung cancer (diagnostic) in HIV infected smokers. In ART-era, lung cancer in HIV infected person appears to have presenting clinical features similar to uninfected person. The effects of HIV infection on lung cancer treatment, including patient's general condition and nutrition, treatment efficacy and toxicity are not well understood. Hence these patients tolerate fewer cycles of chemotherapy than indicated. Moreover, surgical complications are more frequently encountered in HIV infected persons. [24] In the present study all four patients had disseminated disease and could not tolerate the indicated treatment and succumbed within 2-6 months of diagnosis.

NHL - HIV associated NHL presents with extra nodal, bulky and higher stage of disease. Besides cART and anticancer drugs, prophylaxis for Pneumocystis Jirovecii pneumonia and antibiotic prophylaxis for enteric organisms, antiviral and antifungal prophylaxis should be instituted when disease involvement includes more than 2 extra nodal sites. Intrathecal methotrexate or cytosine arabinoside should be administered either prophylactically or in patients with meningeal disease or at high risk of cranial disease by virtue of Burkitt's histology or extensive para nasal sinus (PNS) and base of skull disease. [25] Relapse of lymphoma carries poor prognosis. The median survival is usually less than 1 year. For patients with relapsed B-cell origin lymphoma, a good second line treatment as ESHAP (etopside, methyl prednisolone, cytarabine and cisplatin) should be given. In addition, with significant improvement in HIV control and immune function, autologous stem cell transplantation (ASCT) can be a therapeutic option in selected patients, as is the case in their HIV negative counterparts. [26] In our study, we encountered an extremely rare case of primary skull bone NHL. Only 32 such cases have been reported in the literature till now, and of these only four cases were associated with AIDS. [27-28] Primary osseous NHL of the cranial vault is defined as a solitary mass lesion with no evidence of disease at any other site and no systemic involvement within 6 months of tumor detection. The differential diagnosis includes metastatic carcinoma, meningioma, osteomyelitis, plasma cell tumor, Ewing's sarcoma, histiocytosis and subdural hematoma. Of these tumors $60-90 \%$ is DLBCL, immunoblastic lymphoma, lymphoblastoma or Burkitt's lymphoma. The treatment is surgical excision followed by radiation therapy and chemotherapy $\mathrm{CHOP} / \mathrm{R}-\mathrm{CHOP}$. GI lymphoma is predominant sites $(30-40 \%)$ of all extra nodal lymphoma. The stomach is the most common site followed by small bowel and colorectum. The etiology suggested are Helicobacter pylori infection, hereditary sensitivity to gluten (Coeliac disease), Systemic Lupus Erythematosis, immunosuppression, drugs like thiopurines used in the treatment of irritable bowel syndrome. [29] GI lymphoma constitutes $1-4 \%$ of all GI malignancies, $10-15 \%$ of NHL and $30-40 \%$ of all extra nodal lymphomas. In our department, 24 cases of GI lymphoma were encountered of which one case of Ileocecal junction NHL associated with AIDS was seen. He was treated with 6 cycles of CHOP chemotherapy and showed partial response.

AML is recognized as the most common acute leukemia in adults affecting an estimated $0.5 \%$ of the general population at some point during lifetime with a yearly incidence of 3-5 cases per one lac individuals and a mean age at diagnosis of 68 years. Therefore HIV infection and AML may be considered relatively uncommon disorders in general population. Forghieri F observed only $2 \mathrm{HIV}$ positive patients out of 276 AML patients over a period of 10 years from 2009-2018. [8] The etiology is not well understood till date. The treatment of AML in a patient with well-controlled HIV infection should no longer be considered per se a contraindication to standard intensive chemotherapy for AML and patient's inclusion in clinical trials may certainly help to improve and standardize their clinical management. The allogenic hemopoietic stem cell transplantation is found safe and effective.

Breast: The literature reveals that breast carcinoma does not seem to increase in incidence within the HIV infected population. [30, 31] In present study, all three patients presented with disseminated disease. 
Colorectal Cancer (CRC): O'Neill TJ et al reviewed colo rectal data of 1660 cases and concluded that risk of CRC is similar in people living with and without HIV. [32]

\section{Conclusion}

Epidemiology and the identification of risk factors is a crucial issue, particularly to determine the most appropriate prevention and screening strategies in this population. In the absence of dedicated clinical trials: the cancer management in these patients is based on general recommendations, with specific attention to co-morbidities and drug interactions. PLWH should be treated similar to individuals without HIV infection and should be included in similar clinical trials assuming ART is carefully managed. Dr Suneja G. advised that HIV management during cancer therapy poses many clinical challenges; appropriate cancer treatment can successfully be delivered when oncologist and HIV specialist work together to optimize care of both the diseases. [33] It results in better tolerance to cancer treatment, higher response rates and improved survival. However, modification of HIV therapy may be requested to avoid drug-drug interaction or overlapping toxicities. More frequently CD-4 $\mathrm{T}$-cell counts and viral load testing should be performed during cancer treatment (once a month for the first 3 months then every 3 months thereafter) due to potential drug interactions that makes ART less effective. Since the era of cART, life expectancy of PLWH has improved and is associated with a change in cause of death. In addition, the development of new innovative therapies such as immunotherapy with inhibitory antibodies of immune checkpoint receptors present a new hope for patient care, both infected or not with HIV. In this context, the establishment of the national network CancerVIH makes sense, allowing the establishment of multi-disciplinary consultation meetings involving all the practitioners involved in the care of these patients, as well as the constitution of a national cohort and the promotion of dedicated trials, to improve and optimize the management for these patients. HPV vaccination can potentially prevent cervical and oral cancer thereby HPV vaccination must be recommended not only for young girls but also for boys and HIV infected persons. [17] Follow up of these patients is required to see whether these patients have similar outcomes as compared to sero negative patients of the same stage. Herein in our study we have seen, the cancer if diagnosed in early stage is curable with appropriate treatment and long follow up survival results are evident in present study. In future, effective vaccine against HIV should come.

\section{Conflict of Interest}

There are no conflicts of interest.

\section{Acknowledgements}

Thanks Sangeeta Harivilas and Vijay Laxmi Sharma for assistance in data collection.

\section{References}

[1] Centers for Disease Control (CDC). Update on acquired immune deficiency syndrome (AIDS)--United States. MMWR MorbMortal Wkly Rep 1982; 31 (37): 507-8, 513-4.

[2] Classification system for human T-lymphotropic virus type III/lymphadenopathy-associated virus infections. Centers for Disease Control, U.S. Department of Health and Human Services. Ann Intern Med 1986; 105: 234-7.

[3] Park. Text book of Preventive and Social Medicine, Chapter IV - surface Infections -AIDS. 2019 pp. 371-384.

[4] Zwane NB, Mohangi GU and Shangase SL. Head and Neck Cancers among HIV-positive patients: A five year retrospective study from a Johannesburg hospital, South Africa. South African Dental Journal 2018; 73 (3): 121-126.

[5] Purgina B, Pantanowitz L, and Seethala RR. A Review of Carcinomas Arising in the Head and Neck Region in HIV Positive Patients. Pathology Research International 2011; Article ID 469150, 12 pages doi: 10.4061/2011/469150.

[6] Arnold JD, Byrne ME, Monroe AK and Abbott SE. The risk of anal carcinoma after anogenital warts in adults living with HIV. JAMA Dermatol. 2021; 157 (3): 283-289. doi: 10.1001/jamadermatol.2020.5252.

[7] Re A, Cattaneo C and Rossi G. HIV and lymphoma: from Epidemiology to clinical management. Mediterranean $\mathrm{J}$ of Hemato \& Infectious Diseases. 2019; 11: e 2019004.

[8] Forghieri F, Nasillo V, Bettelli F et al. Review Acute Myeloid Leukemia in Patients Living with HIV Infection: Several Questions, Fewer Answers. Int. J. Mol. Sci. 2020; 21: 1081; doi: 10.3390/ijms21031081.

[9] Barnardt $P$ and Relling $M$. Gestational Trophoblastic Neoplasm and Women Living With HIV and/or AIDS. South African Journal HIV Med. 2015; 16 (1): 344.

[10] Rachel A. Ignacio B, Lin LL, Rajdev L and Chiao E. Evolving Paradigms in HIV Malignancies: Review of Ongoing Clinical Trials. J National Comprehensive Cancer Network 2018; 16 (8): 1018-1026.

[11] R K Tanwar, Bhati R, Soni N, Dangayach SK and Saxena B. Situs Inversus Totalis associated with Cancer: Report of three cases and Review of literature. Medical Journal Dr D Y Patil Vidhyapeeth. 2019; 12 (2): 154-168.

[12] Sonkaria M, Tanwar RK, Saxena B, Vashishtha N, Singh RK. Primary breast lymphoma: A case series and review.. Indian $\mathrm{J}$ Case Reports. 2020; 6 (6): 288-291.

[13] Gupta AK, Li B, Cerniglia GJ, Ahmed MS, Hahn SM and Maity A. The HIV Protease Inhibitor Nelfinavir Downregulates Akt Phosphorylation by Inhibiting Proteasomal Activityand Inducing the Unfolded Protein Response. Neoplasia 2007; 9 (4): 271-278.

[14] Lin A and Maity A. Molecular Pathways: A Novel Approach to Targeting Hypoxia and Improving Radiotherapy Efficacy via Reduction in Oxygen Demand. Clinical Cancer Research; 2015; 21 (9): 1995-2000. 
[15] Nayyar SS, Thiagarajan S, Malik A, et al. Head and neck squamous cell carcinoma in HIV, HBV and HCV seropositive patients - Prognosis and its predictors. Journal of Cancer Research and Therapeutics 2020; 16 (3): 619-23.

[16] Ceccarelli M, Rullo EV, Facciolà A, et al. Head and neck squamous cell carcinoma and its correlation with human papillomavirus in people living with HIV: a systematic review. Oncotarget, 2018; 9 (24): 17171-17180.

[17] D'Andrea F, Pellicano GF, Rullo EV et al. Cervical Cancer in women living with HIV: A review of the literature. World Cancer Research Journal 2019; 6: e 1224.

[18] Grover S, Mehta P, Wang Q et al. Association between CD-4 count and chemo radiation therapy outcomes among cervical cancer patients with HIV. Journal of Acquired Immune deficiency Syndromes: 2020; 85 (2): 201-208.

[19] Shrivastava SK, Engineer R, Rajadhyaksha S and Dinshaw KA. HIV infection and invasive cervical cancers, treatment with radiation therapy: Toxicity and outcome (Green Journal). DOI: https://doi.org/10.1016/J.Radonc. 2004; 11.006.

[20] Levinson KL, Riedel DJ, Ojalvo LS et al. Gynecologic Cancer in HIV infected women: Treatment and outcomes in a multiinstitutional cohort. AIDS 2018; 32 (2): 171-177.

[21] Ashley I. Choriocarcinoma in a patient with human immunodeficiency virus: case presentation and review of the literature. The Mount Sinai Journal of Medicine, New York 2002; 69 (5): 334-337.

[22] Tanwar RK, Saxena B, Garg R, Goyal H, Deepak D. Gestational Trophoblastic Neoplasia: A Retrospective Study of 23 Cases. Cancer Research Journal 2020; 8 (3): 45-50.

[23] Sigel K, Makinson A and Thaler J. Lung Cancer in Persons with HIV. Curr Opin HIV AIDS. 2017; 12 (1): 31-38. doi: $10.1097 / \mathrm{COH} .0000000000000326$.

[24] Hooker CM, Meguid RA, Hulbert A et al. Human immunodeficiency virus infection as a prognostic factor in surgical patients with non- small cell lung cancer. Ann Thorac Surg 2012; 93: 405-412.

[25] Powel T, Matthews G and Bower M. AIDS related systemic non-Hodgkin's lmphoma. Sexually Transmitted Infections 2000; 76: 335-341.

[26] Oishi N, Bagan JV, Javier K and Zapater E. Head and Neck Lymphoma in HIV Patients: A Clinical Perspective. Int. Arch Otorhinolaryngol 2017; 21:300-407.

[27] Huang J, Tang D, Xu Y, Wang X, Yu C and Dang Y. Head trauma complicated with primary cranial vault lymphoma. A case report. Medicine 2019; 98: 7 (e 14465).

[28] Choudhary P, Kalwaniya S, Agarwal L and Saxena S. Primary multifocal skull lymphoma in an acquired immunodeficiency syndrome patient. West African Journal of Radiology 2015; 22 (2): 107-109.

[29] Thomas AS, Schwartz M and Quigley E. Gastrointestinal Lymphoma: the new mimic. BMJ Open Gastroenterology 2019; 6: e000320.

[30] Palan M, Shousha S, Krell J, and Stebbing J. Case Report. Breast Cancer in the Setting of HIV. Pathology Research International Volume 2011; Article ID 925712: 4 pages. Doi: $10.4061 / 2011 / 925712$.

[31] Chirkut S. Breast cancer, human immunodeficiency virus and highly active antiretroviral treatment; implications for a highrate seropositive region. Oncology Reviews 2019; 13: 376.

[32] O'Neill TJ, Nguemo JD, Tynan AM, Burchell AN and Antoniou T. Risk of Colorectal Cancer and Associated Mortality in HIV: A Systematic Review and Meta-Analysis. J Acquir Immune Defic Syndr 2017; 75: 439-447.

[33] Suneja G. New NCCN Guidelines: Cancer Management in People Living With HIV. J National Comprehensive Cancer Network 2018; 16 (5.5): 597-599. 